\title{
Practices and Attitudes Regarding Women Undergoing Fertility Preservation: A Survey of the National Physicians Cooperative
}

\author{
Pietro Bortoletto, MD, ${ }^{1}$ Rafael Confino, BS, ${ }^{2, *}$ Brigid M. Smith, CCRP, \\ Teresa K. Woodruff, $\mathrm{PhD}^{3}$, and Mary Ellen Pavone, $\mathrm{MD}, \mathrm{MSCl}^{2}$
}

Purpose: To describe physician attitudes and variations in oncofertility treatment strategies.

Methods: An exploratory online survey administered between December 1, 2014 and January 27, 2015 to 185 members of the National Physicians Cooperative (NPC).

Results: Twenty-eight percent (52 of 185) of NPC members responded to the online survey. Fifty percent of respondents were obstetrician-gynecologists working largely in academic medical centers. Thirty-eight percent stated that 14 was the youngest age they felt comfortable performing oocyte retrievals with $35 \%$ stating that any age was acceptable as long as they were postpubertal. Short stimulation protocols, utilizing a gonadotropin-releasing hormone $(\mathrm{GnRH})$ antagonist $(86 \%)$, were more common than long stimulation or microdose Lupron flare protocols (23\% and $18 \%$ ), respectively, which utilize a GnRH agonist. Random start protocols were used by $77 \%$ and over $90 \%$ perform luteal phase starts. When using random start protocols, $64 \%$ use gonadotropins only and 32\% start GnRH antagonists alone for several days before starting gonadotropins. Fifty-five percent of physicians were comfortable stimulating ovarian cancer patients only after clearance from an oncologist. Aromatase inhibitors $(77 \%)$ were significantly more common than tamoxifen $(24 \%)$ for stimulation in breast cancer patients $(p=0.0006)$. When considering ovarian stimulation after chemotherapy, 24\% expressed comfort only if blood counts are normal, $38 \%$ tend to wait 3 months. Regarding experimental treatment, $83 \%$ report discussing the use of $\mathrm{GnRH}$ agonists alone and $64 \%$ of clinics offer ovarian tissue cryopreservation.

Conclusions: This study underlines the wide variation that exists in stimulation and phase start techniques, patient selection, comfort levels, and cancer type-specific decision making.

Keywords: oncofertility, fertility preservation, National Physicians Cooperative, cancer

\section{Introduction}

T He American Cancer Society estimates that there were 1.6 million new cases of cancer diagnosed in the United States in 2015, with more than 86,000 of those being in women of reproductive age. At the same time, disease-specific 5 -year survival has continued to increase, from $49 \%$ in the $1970 \mathrm{~s}$ to $68 \%$ in the mid 2000 s. ${ }^{1}$ While many of these patients have been able to overcome a diagnosis of cancer, their next hurdle is managing life after cancer. It is well known that several chemotherapeutic agents and radiation treatments have detrimental effects on ovarian reserve. ${ }^{2}$ The term oncofertility was coined to describe a medical subspecialty dedicated to preserving future fertility in light of gonadotoxic cancer treatment. ${ }^{3}$ Oncofertility refers to the integrated network of clinical resources to develop methods of preserving or restoring reproductive capabilities in patients diagnosed with cancer. ${ }^{4}$ Oncologists are increasingly called upon to inform their patients of the reproductive consequences of cancer treatment and refer their patients to fertility specialists to optimize their reproductive options post-treatment. Furthermore, fertility preservation (FP) consultation has been shown to reduce long-term regret

\footnotetext{
${ }^{1}$ Department of Obstetrics and Gynecology, Brigham and Women's Hospital, Boston, Massachusetts.

${ }^{2}$ Division of Reproductive Endocrinology and Infertility, Department of Obstetrics and Gynecology, Northwestern University Feinberg School of Medicine, Chicago, Illinois.

${ }^{3}$ Division of Reproductive Science in Medicine, Department of Obstetrics and Gynecology, Northwestern University Feinberg School of Medicine, Chicago, Illinois.

*Co-first author.
} 
and dissatisfaction and is associated with improved quality of life in female cancer patients. ${ }^{5}$

Unfortunately, FP options vary widely based on age, cancer type and location, and proposed chemotherapy and/or radiation treatment regiments. For postreproductive aged women, there is an added incentive to preserve reproductive organs to sustain hormonal balance before the natural onset of menopause. ${ }^{6-8}$ Adolescent and young adult cancer survivors have identified the risks of treatment-related infertility as important, and up to $75 \%$ of women in this age group are interested in the possibility of having children after treatment. ${ }^{9}$ However, there are also ethical and technical considerations that add another layer of complexity to decision making for both the patient and physicians involved. ${ }^{10}$ Many of these remain unresolved or under-reported.

The Oncofertility Consortium is an interdisciplinary initiative funded by the National Institutes of Health to study the issue of FP and provide education to patients and providers alike. ${ }^{4,11}$ The National Physicians Cooperative (NPC), a network organized by the Oncofertility Consortium, consists of physicians (MD or DO), patient navigators, research coordinators, and research scientists $(\mathrm{PhD})$ dedicated to preserving fertility of their patients. The goal of the present survey study was to describe the wide variety of attitudes and practice patterns of obstetrician-gynecologist members of the NPC concerning the care of oncofertility patients.

\section{Methods}

An online survey administered through SurveyMonkey (Palo Alto, CA) was distributed to 185 members of the NPC between December 1, 2014 and January 27, 2015. The NPC is a network of 99 physicians (MD or DO) members and 86 nonclinical members (e.g., patient navigators, research coordinators, and research scientists). NPC members were asked 28 multiple-choice questions pertaining to their practice environment, treatment strategies, and comfort-level concerning the care of oncofertility patients. The goal response rate for this online questionnaire was $30 \%$ for physician members. Only physician respondents were included in the final analysis given their area of expertise and ability to accurately answer the clinical questions this survey was focused on. Respondents were not required to answer every survey question. SPSS Statistics 20 for Windows (IBM Corporation, Armonk, NY) was used to calculate Pearson's chi-square at $95 \%$ confidence interval. The Northwestern University Institutional Review Board (IRB) considered this study exempt of IRB approval.

\section{Results}

Fifty-two of 185 members of the NPC responded to the online questionnaire. Of the 52 respondents, 26 (50\%) were physician members who worked in academic institutions $(65 \%)$ as board certified reproductive endocrinologists (73\%). Forty-two percent reported working in a practice of two to four members with $37 \%$ in a practice of five to nine members. Seventy-two percent had been in practice more than 5 years (Table 1). Before 2007, a majority of physicians reported caring for $0-9$ patients per year for FP $(68 \%)$, but by 2013 a majority saw more than 9 patients per year for FP $(68 \%)$. In $2013,68 \%$ of clinics saw fewer than nine patients per year under 18 years old. When performing oocyte retrievals on young patients, $38 \%$ reported that 14 years old was the youngest age they felt comfortable, while $35 \%$ felt comfortable at any age as long as they were postpubertal. Fifty-four percent had a children's hospital affiliated with their clinic and $83 \%$ had access to a reproductive urologist for consultation. Additionally, $54 \%$ did not use a patient navigator, which refers to a person who coordinates patient care, connects patients with resources, and helps patients understand the healthcare system, and $74 \%$ had optional psychological counseling available. Eighty-seven percent of respondents surveyed indicated that their clinic offered social FP, meaning oocyte retrieval and storage for nonmedical indications. When performing retrievals, $91 \%$ utilized conscious sedation alone for anesthesia and $41 \%$ batched cycles to allow for ease of cycle monitoring and retrieval.

When performing controlled ovarian stimulation, short protocols $(86 \%)$, which use gonadotropin-releasing hormone $(\mathrm{GnRH})$ antagonist to prevent premature ovulation, were more popular than long protocols $(23 \%)$ or microdose Lupron flare protocols $(18 \%)$, which both use GnRH agonist at different times to prevent premature ovulation. Seventy-seven percent reported using random start protocols, where a patient can be stimulated on presentation regardless of the menstrual-cycle phase (Fig. 1A). When performing random stimulation starts, $64 \%$ start gonadotropins only, regardless of which phase patient is currently in. In regards to cycle phase, $91 \%$ of clinics perform luteal phase starts on FP patients (Fig. 1B). For luteal phase starts, $31 \%$ utilize GnRH antagonists alone for several days before starting gonadotropins and the minority (18\%) start $\mathrm{GnRH}$ antagonists and gonadotropins at the same time. When considering ovulation triggers, which initiate oocyte release from the ovary, for FP patients, $41 \%$ reported using a GnRH agonist "always" and 50\% "sometimes," when worried about ovarian hyperstimulation syndrome (OHSS). GnRH agonists provide a large luteinizing hormone ( $\mathrm{LH})$ surge to stimulate oocyte release but the effect on LH levels is short lived, minimizing the risk of OHSS.

When considering clinical practice patterns, 55\% felt comfortable stimulating and retrieving patients with ovarian cancer after clearance from a gynecologic oncologist, while $32 \%$ did not, regardless of clearance $(p=0.003)$. When considering the different subtypes of ovarian cancer, $46 \%$ felt comfortable stimulating and retrieving patients with epithelial ovarian cancer of low malignant potential or borderline tumors, $23 \%$ for sex cord stromal tumors, and $18 \%$ for germ cell tumors (Fig. 2). Only 5\% felt comfortable with stimulating patients with high-grade serous carcinoma of the ovary. When stimulating breast cancer patients seeking FP, 77\% reported using aromatase inhibitors such as letrozole or anastrazole for ovarian stimulation while only $27 \%$ used tamoxifen ( $p=0.0004$ ) (Fig. 1C, D).

Lastly, when asked about attitudes toward performing ovarian stimulation and oocyte retrievals after chemotherapy exposure, $24 \%$ expressed comfort only if blood counts are normal (without evidence of thrombocytopenia, neutropenia, or severe anemia), 38\% tend to wait 3 months, and 5\% do not feel comfortable at all. Eighty-three percent reported discussing the use of GnRH agonists alone as a method for FP, although an experimental technique. Ovarian tissue cryopreservation was offered at $64 \%$ of clinics with an additional $18 \%$ in the process of IRB submission for an open protocol. 
Table 1. Demographic Characteristics of SuRvey Respondents

Respondent answers
Primary role in the IVF clinic
Physician
Other
What type of setting best describes your practice?
Academic
Private practice
Other
How many physicians are in your practice?
1
$2-4$
$5-9$
10 or more
If you are a physician, are you board certified?
Yes, in reproductive
endocrinology and
infertility
Yes, in obstetrics and
gynecology only
No, but board
eligible
Yes, in other
specialty

How many years have you been in practice since finishing fellowship?

2-4

$5-9$

10 or more

$8(33.3)$

Not applicable

$26(50)$

$26(50)$

$17(65.4)$

7 (26.9)

2 (7.7)

$3(11.5)$

$11(42.3)$

$9(34.6)$

$3(11.5)$

$19(73.1)$

Before 2007, approximately how many patients a year did you see for fertility preservation for a medical condition?

None

$10(41.7)$

Less than 9

$6(25)$

$10-25$

4 (16.7)

25 or more

$1(4.2)$

Unknown

$3(12.5)$

Last year (2013), how many fertility preservation patients did you see for a medical condition (e.g., cancer, lupus, etc.)?

0

$3(12.5)$

Less than 9

$10-24$

$3(12.5)$

25 or more

$11(45.8)$

$7(29.2)$

Last year (2013), how many patients did you see in your clinic who are under 18 years of age?

0

Less than 9

$10-24$

$6(25)$

$11(45.8)$

$3(12.5)$

$4(16.7)$

Is your IVF clinic affiliated with a Children's Hospital?

Yes

$13(54.2)$

No

$8(33.3)$

Not applicable

$3(12.5)$

Is your IVF clinic partnered with a reproductive urologist?

Yes-we have one

on staff as part of our

REI practice
TABLE 1. (CONTINUED)

\begin{tabular}{lc}
\hline Respondent answers & $\mathrm{N}(\%)$ \\
\hline $\begin{array}{l}\text { Yes-we are able to } \\
\text { easily refer to a } \\
\text { reproductive } \\
\text { urologist }\end{array}$ & $11(45.8)$ \\
No & \\
\end{tabular}

Does your clinic work with personnel (patient navigators)

to help patients who seek fertility preservation for medical conditions efficiently receive information and care?

Yes

$11(45.8)$

No

$13(54.2)$

Does your clinic offer IVF patients counseling with a psychologist

Yes-mandatory

Yes-optional

$17(74)$

No

Does your clinic offer social fertility preservation

(e.g., IVF for women who want to delay

child-bearing)?

Yes

No

$3(13)$

IVF, in vitro fertilization; REI, reproductive endocrinology and infertility.

\section{Conclusion}

Quality cancer care includes discussions about fertility and referrals to fertility specialists for patients at risk for sterility. Despite this, few NCI-designated comprehensive cancer centers seem to be following ASCO guidelines to ensure provision of timely information and referrals to reproductive age patients. ${ }^{12}$ Multiple barriers to referral for FP have been reported, including lack of time, lack of knowledge, and poor prognosis. A study by Forman et al. found that while $82 \%$ of oncologists have at some point referred at least one patient for fertility consultation, nearly $50 \%$ rarely do so. ${ }^{13}$ With the increasing number of reproductive age cancer survivors, the need for reliable, thoughtful, and evidence-based information regarding FP referral and treatment is greater than ever.

In our study, which largely consisted of academic reproductive endocrinologists, a substantial increase in oncofertility volume was noted in just over 5 years' time. Still, practice variations exist that may negatively impact access to care. While most clinicians report seeing patients under the age of 18 , only $38 \%$ felt comfortable managing patients as young as 14 years of age. In prepubertal patients without a functioning hypothalamic-pituitary-ovarian (HPO) axis, ovarian stimulation cannot be performed. However, investigational techniques such as in vitro maturation of immature oocytes, ovarian tissue cryopreservation, and pharmacologic preservation using $\mathrm{GnRH}$ are all emerging therapies that may prove useful in preserving fertility for childhood cancer survivors. ${ }^{14}$ Sixty-four percent of physicians that answered our survey have an open protocol for ovarian tissue cryopreservation and $91 \%$ talk to their patients about using a GnRH agonist alone as an experimental approach to FP.

Depending on the type of cancer a patient presented with, comfort levels and practice patterns differed substantially. Up to $32 \%$ did not feel comfortable stimulating and retrieving 

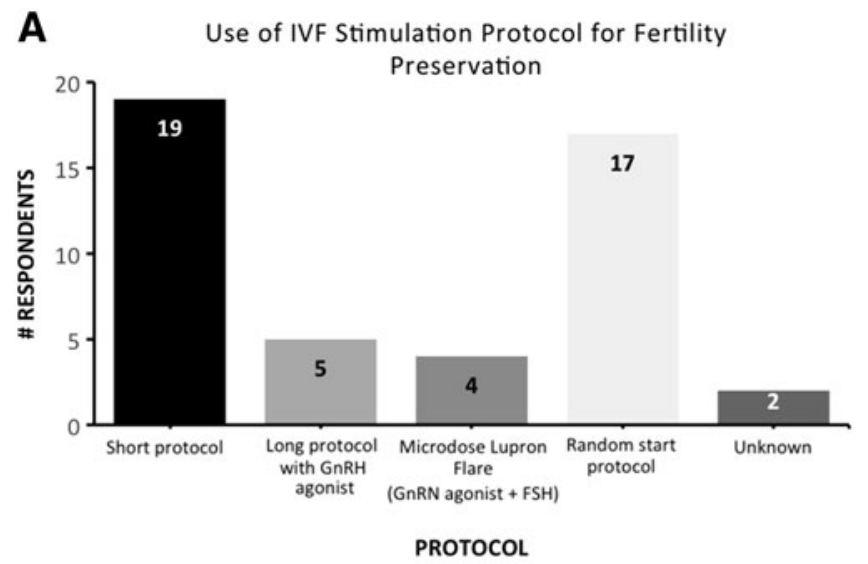

C

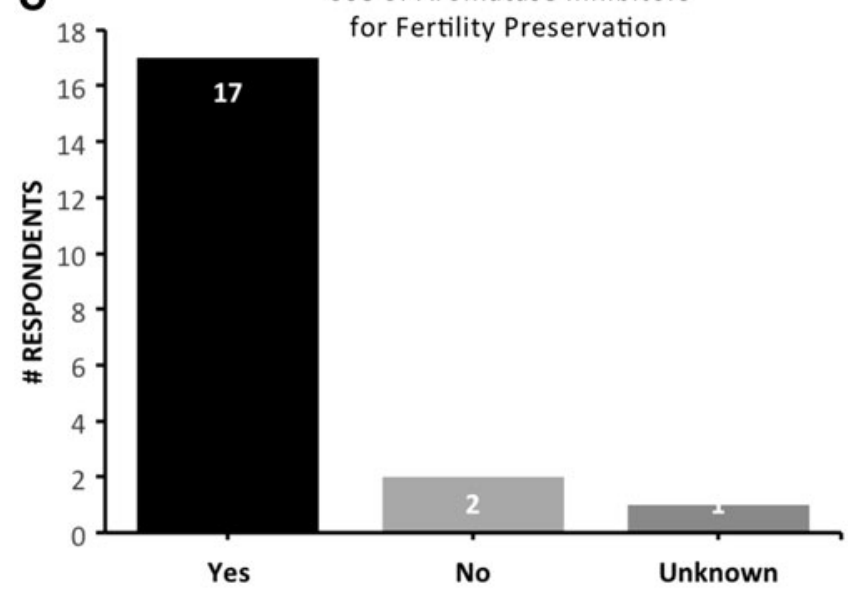

B
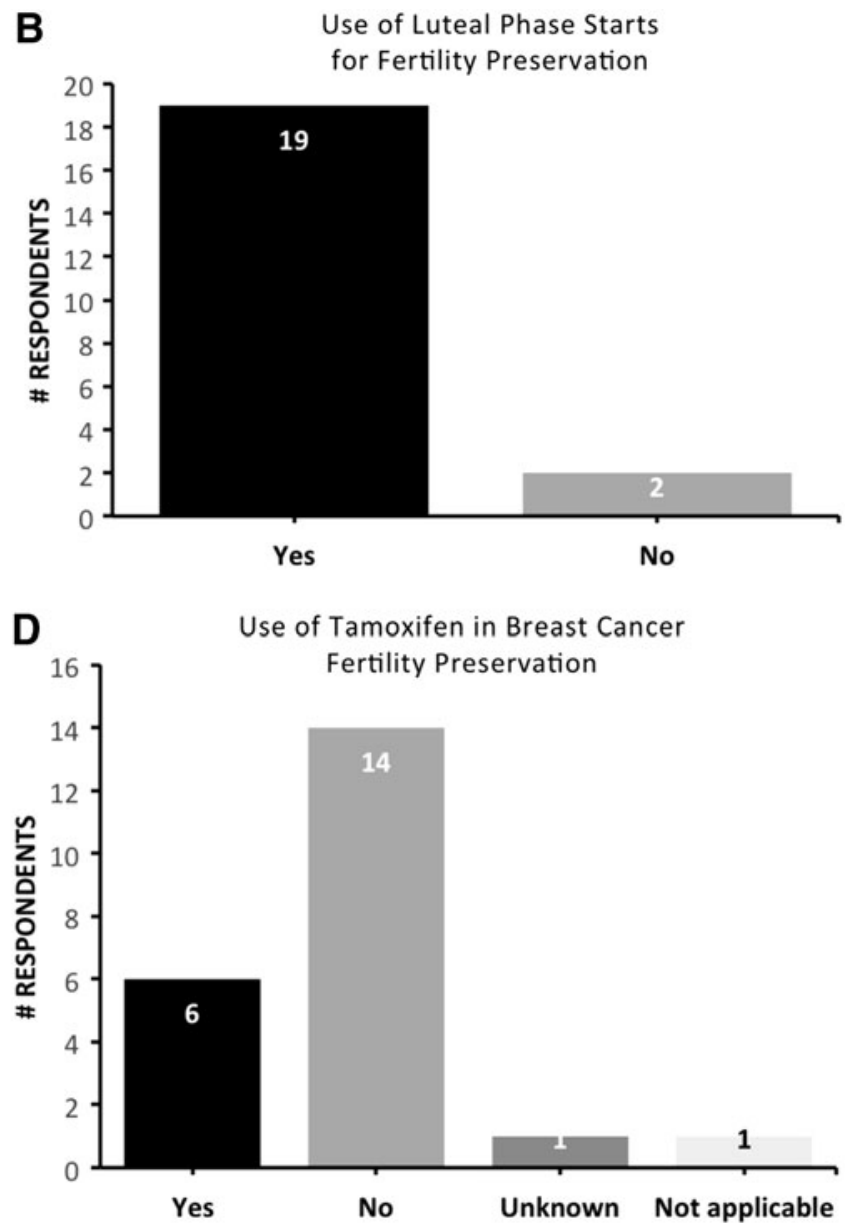

FIG. 1. Bar graphs representing respondent answers to the following: (A) what IVF stimulation protocols do you use for fertility preservation, (B) do you perform luteal phase starts, $(\mathbf{C})$ when stimulating breast cancer fertility preservation patients, do you use aromatase inhibitors? (D) when stimulating breast cancer fertility preservation patients, do you use Tamoxifen? IVF, in vitro fertilization.

patients with ovarian cancer, despite clearance from an oncologist, and of those that did feel comfortable, less than 5\% felt comfortable doing so if they were diagnosed with a highgrade serous carcinoma of the ovary. According to the American College of Obstetrics and Gynecology (ACOG), a small proportion of young women with ovarian cancer are eligible for fertility-sparing surgery, namely stage IA and nonclear cell histology grade 1 or $2 .{ }^{15}$ For women with stages II-III ovarian cancer, recurrence and mortality rates after fertility-sparing surgery were as high as $42.8 \%$ and $23.8 \%$,
FIG. 2. Bar graph representing respondent answers to "What types of ovarian cancer are you comfortable stimulating and retrieving?"

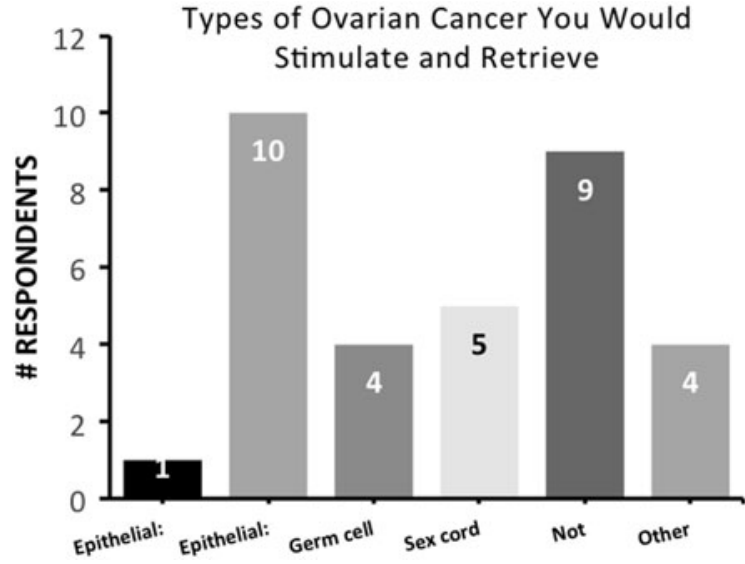

Epithelial: high grade serous carcinoma

Epithelial: low malignant potential/borderline

- Germ cell (dysgerminoma, endodermal sinus and choriocarcinoma) Sex cord stromal (granulosa, granulosa-theca, SertoliLeydig)

not applicable 
respectively, with obstetric outcomes reported in $14.2 \%$ of cases. For these women, radical surgical treatment remains the standard of care. ${ }^{16}$

When looking at FP in breast cancer patients, the vast majority reported using aromatase inhibitors over tamoxifen $(77 \%$ vs. $27 \%, p=0.0004$ ) for ovarian stimulation. This likely represents the belief that a minimal increase in serum estrogens with letrozole is a safer approach for these patients with estrogen receptive cancer. Interestingly, Novartis, the manufacture of letrozole, does not recommend the use of this drug as an inducer of ovulation due to the potential risk of harm to mother and fetus, despite studies to suggest it is safe. ${ }^{17}$ Notwithstanding, embryo cryopreservation after ovarian stimulation with the letrozole and follicle-stimulating hormone protocol has been shown to preserve fertility in women with breast cancer and results in live birth rate per embryo transfer comparable to those expected in a noncancer population undergoing in vitro fertilization $(45 \%$ vs. $38.2 \%, p=0.2) .{ }^{18}$

Regardless of cancer diagnosis, physicians were divided on when to stimulate patients after chemotherapy exposure, with $24 \%$ waiting until blood counts normalized, and $38 \%$ waiting at least 3 months, an arbitrary time cut off. These differences in comfort and practice pattern are likely multifactorial. Lack of evidence-based guidelines probably serve as a deterrent to reproductive endocrinologists who do not wish to interfere with or alter the natural course of a patient's disease or cancer treatment by using gonadotropic drugs. It is, however, known that women with cancer who are exposed to chemotherapy have lower baseline antral follicle counts and should expect a lower number of oocytes retrieved after ovarian stimulation for FP, compared with healthy, agematched patients but the pregnancy outcomes are similar to chemotherapy-naïve patients if they reach retrieval. ${ }^{19,20}$

With regards to ovarian stimulation protocols and phase starts, again a wide variety of patterns emerged. Ninety-one percent perform luteal phase starts with $64 \%$ utilizing gonadotropins only, regardless of phase. The additions of pre-treating with GnRH antagonists or utilizing GnRH antagonists plus gonadotropins concurrently appeared to be a less common option. Dragisic et al. have previously described the use of pre-treatment with GnRH antagonists and estradiol patch may improve ovarian responsiveness during stimulation and lead to more uniform follicular development, more oocytes retrieved, and improved pregnancy rates. ${ }^{21}$ There are, unfortunately, few studies examining the use of pre-treating or co-treating FP patients with GnRH antagonists, and so it is unclear how beneficial this would be.

The goal of this study was to specifically describe practice patterns and attitudes concerning the care of oncofertility patients among a self-selected group of physician members of the NPC. The strengths of our study include the fact that, we asked a wide range of questions that describe in detail stimulation strategies, drug protocols, and comfort levels with different cancer subtypes. Additionally, we were able to describe the practice environment and patient volumes that NPC providers are seeing over the last several years. Our online survey is not without its limitations. First, we surveyed members of the NPC, an organization in which FP practitioners from around the country self-enroll. The results likely represent the "best case scenario" from high volume centers with physicians who have experience providing oncofertility care. Second, we specifically sought to analyze physician responses and excluded responses from mid-level providers and ancillary staff. This likely leads to a self-serving and selective-perception bias. Lastly, our response rate of $28 \%$ of all NPC members, but $50 \%$ of physician members, may not completely and accurately describe oncofertility care that is occurring in every part of the United States.

It is crucial for oncologists and reproductive endocrinologists to partner in standardizing the practice of referral and treatment for oncofertility care. Fertility consultation has been shown to reduce long-term regret and dissatisfaction and is associated with improved quality of life in female cancer patients. ${ }^{5}$ This study provides an estimation of the current physician practices, attitudes, and behaviors concerning the care of oncofertility patients. Our survey underlines the wide variation that exists in stimulation and phase start techniques, patient selection and comfort levels, and cancer type-specific decision making. The development of comprehensive recommendations and guidelines for reproductive endocrinologists and infertility physicians is necessary to meet the expectations and fertility needs of cancer patients.

\section{Acknowledgments}

The authors would like to thank the NIH Specialized Research Center (P50) for their generous support of the Oncofertility Consortium and National Physicians Cooperative. This research study is partially supported by the Center for Reproductive Health After Disease (P50HD076188) from the National Institutes of Health National Center for Translational Research in Reproduction and Infertility (NCTRI).

\section{Author Disclosure Statement}

No competing financial interests exist.

\section{References}

1. American Cancer Society. Cancer facts \& figures 2016. Atlanta: American Cancer Society; 2016.

2. Jeruss JS, Woodruff TK. Preservation of fertility in patients with cancer. N Engl J Med 2009;360(9):902-11.

3. Woodruff TK. The emergence of a new interdiscipline: oncofertility. Cancer Treat Res 2007;138:3-11.

4. Woodruff TK. The Oncofertility Consortium-addressing fertility in young people with cancer. Nat Rev Clin Oncol 2010;7(8):466-75.

5. Deshpande NA, Braun IM, Meyer FL. Impact of fertility preservation counseling and treatment on psychological outcomes among women with cancer: a systematic review. Cancer 2015;121(22):3938-47.

6. Donnez J, Silber S, Anderson CK, et al. Children born after autotransplantation of cryopreserved ovarian tissue. a review of 13 live births. Ann Med 2011;43(6):437-50.

7. Silber S, Kogawa N, Kuwbyama M, Gosden R, et al. Duration of fertility after fresh and frozen ovary transplantation. Fertil Steril 2010;94(6):2191-6.

8. Diitrich R, Lotz L, Keck G, et al. Live birth after ovarian tissue autotransplantation following overnight transportation before cryopreservation. Fertil Steril 2012;97(2):387-90.

9. Geue K, Richter D, Schmidt R, et al. The desire for children and fertility issues among young German cancer survivors. J Adolesc Health 2014;54(5):527-35. 
10. Tercyak K, Mays D, Johnson A, et al. Oncofertility and quality of life among adolescent and young adult survivors of childhood cancer. J Clin Oncol 2016;34(3 Suppl):222.

11. Backhus LE, Kondapalli LA, Chang RJ, et al. Oncofertility consortium consensus statement: guidelines for ovarian tissue cryopreservation. Cancer Treat Res 2007;138:235-9.

12. Clayman ML, Harper MM, Quinn GP, et al. Oncofertility resources at NCI-designated comprehensive cancer centers. J Natl Compr Cancer Netw 2013;11(12):1504-9.

13. Forman EJ, Anders CK, Behera MA. A nationwide survey of oncologists regarding treatment-related infertility and fertility preservation in female cancer patients. Fertil Steril 2010;94(5):1652-6.

14. Trudgen K, Ayensu-Coker L. Fertility preservation and reproductive health in the pediatric, adolescent, and young adult female cancer patient. Curr Opin Obstet Gynecol 2014;26(5):372-80.

15. American College of Obstetricians and Gynecologists. ACOG Practice Bulletin. Management of adnexal masses. Obstet Gynecol 2007;110(1):201-14.

16. Petrillo M, Legge F, Ferrandina G, et al. Fertility-sparing surgery in ovarian cancer extended beyond the ovaries: a case report and review of the literature. Gynecol Obstet Invest 2013;77(1):1-5.

17. Munoz E, Gonzalez N, Munoz L, et al. Ovarian stimulation in patients with breast cancer. Ecancermedicalscience 2015;9:504.
18. Oktay K, Turan V, Bedoschi G, et al. Fertility preservation success subsequent to concurrent aromatase inhibitor treatment and ovarian stimulation in women with breast cancer. J Clin Oncol 2015;33(22):2424-9.

19. Friedler S, Koc O, Gidoni Y, et al. Ovarian response to stimulation for fertility preservation in women with malignant disease: a systematic review and meta-analysis. Fertil Steril 2012;97(1):125-33.

20. Chan JL, Johnson LNC, Efymow BL, et al. Outcomes of ovarian stimulation after treatment with chemotherapy. J Assist Reprod Genet 2015;32(10):1537-45.

21. Dragisic KG, Davis OK, Fasouliotis SJ, Rosenwaks Z, et al. Use of a luteal estradiol patch and a gonadotropin-releasing hormone antagonist suppression protocol before gonadotropin stimulation for in vitro fertilization in poor responders. Fertil Steril 2005;84(4):1023-6.

Address correspondence to: Mary Ellen Pavone, MD, MSCI Division of Reproductive Endocrinology and Infertility Department of Obstetrics and Gynecology Northwestern University Feinberg School of Medicine 633 North Street Clair Avenue

Suite 18-027

Chicago, IL 60611

Email: m-pavone@ northwestern.edu 